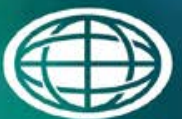

Savannah River

National Laboratory ${ }^{m}$

OPERATED BY SAVANNAH RIVER NUCLEAR SOLUTIONS

\title{
A Basis for Modifying the Tank 12 Composite Sampling Design (U)
}

\section{E.P. Shine}

November 2014

SRNL-STI-2014-00551, Revision 1 


\section{DISCLAIMER}

This work was prepared under an agreement with and funded by the U.S. Government. Neither the U.S. Government or its employees, nor any of its contractors, subcontractors or their employees, makes any express or implied:

1. warranty or assumes any legal liability for the accuracy, completeness, or for the use or results of such use of any information, product, or process disclosed; or

2. representation that such use or results of such use would not infringe privately owned rights; or

3. endorsement or recommendation of any specifically identified commercial product, process, or service.

Any views and opinions of authors expressed in this work do not necessarily state or reflect those of the United States Government, or its contractors, or subcontractors.

\section{Printed in the United States of America}

Prepared for U.S. Department of Energy 
Keywords: Closure, Tank Farm, Tank 12, Composite Sampling

Retention: Permanent

\title{
A Basis for Modifying the Tank 12 Composite Sampling Design (U)
}

\author{
E. P. Shine
}

November 24, 2014

Prepared for the U.S. Department of Energy under

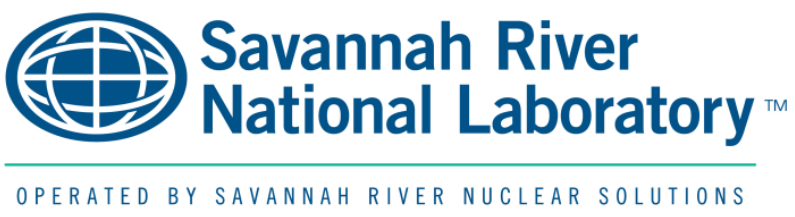
contract number DE-AC09-08SR22470. 


\section{REVIEWS AND APPROVALS}

AUTHOR:

E. P. Shine, Author, Analytical Development

Date

TECHNICAL REVIEW:

APPROVAL: 
SRNL-STI-2014-00551

Revision 1

This Page Intentionally Left Blank 


\section{EXECUTIVE SUMMARY}

The SRR sampling campaign to obtain residual solids material from the Savannah River Site (SRS) Tank Farm Tank 12 primary vessel resulted in obtaining appreciable material in all 6 planned source ${ }^{1}$ samples from the mound strata but only in 5 of the 6 planned source samples from the floor stratum. Consequently, the design of the compositing scheme presented in the Tank 12 Sampling and Analysis Plan, Pavletich (2014a), must be revised.

Analytical Development of SRNL statistically evaluated the sampling uncertainty associated with using various compositing arrays and splitting one or more samples for compositing. The variance of the simple mean of composite sample concentrations is a reasonable standard to investigate the impact of the following sampling options.

Composite Sample Design Option (a). Assign only 1 source sample from the floor stratum and 1 source sample from each of the mound strata to each of the composite samples. Each source sample contributes material to only 1 composite sample. Two source samples from the floor stratum would not be used.

Composite Sample Design Option (b). Assign 2 source samples from the floor stratum and 1 source sample from each of the mound strata to each composite sample. This infers that one source sample from the floor must be used twice, with 2 composite samples sharing material from this particular source sample. All five source samples from the floor would be used.

Composite Sample Design Option (c). Assign 3 source samples from the floor stratum and 1 source sample from each of the mound strata to each composite sample. This infers that several of the source samples from the floor stratum must be assigned to more than one composite sample. All 5 source samples from the floor would be used.

Using fewer than 12 source samples will increase the sampling variability over that of the Basic Composite Sample Design, Pavletich (2013). Considering the impact to the variance of the simple mean of the composite sample concentrations, the recommendation is to construct each sample composite using four or five source samples. Although the variance using 5 source samples per composite sample (Composite Sample Design Option (c)) was slightly less than the variance using 4 source samples per composite sample (Composite Sample Design Option (b)), there is no practical difference between those variances. This does not consider that the measurement error variance, which is the same for all composite sample design options considered in this report, will further dilute any differences. Composite Sample Design Option (a) had the largest variance for the mean concentration in the three composite samples and should be avoided.

These results are consistent with Pavletich (2014b) which utilizes a low elevation and a high elevation mound source sample and two floor source samples for each composite sample. Utilizing the four source samples per composite design, Pavletich (2014b) utilizes aliquots of Floor Sample 4 for two composite samples.

\footnotetext{
${ }^{1}$ In this report a source sample is a sample of residual material obtained directly from the primary vessel of Tank 12 . This contrasts with a composite sample which is not obtained directly from Tank 12, but receives aliquots of residual material from some of the source samples.
} 


\section{TABLE OF CONTENTS}

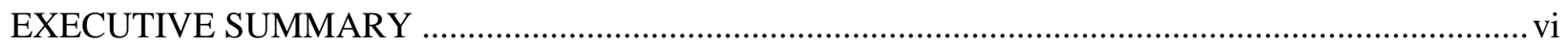

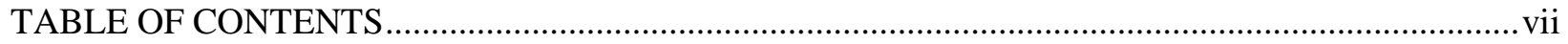

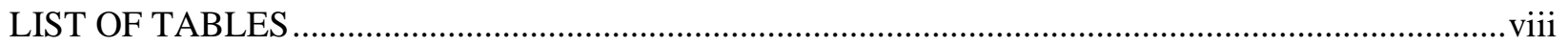

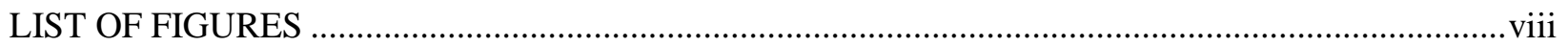

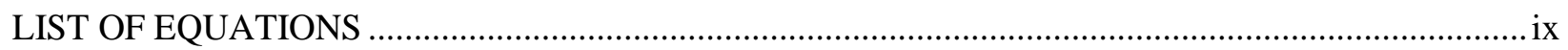

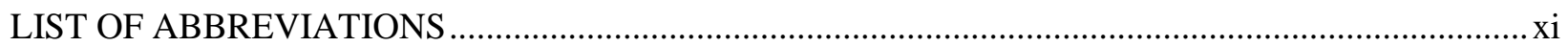

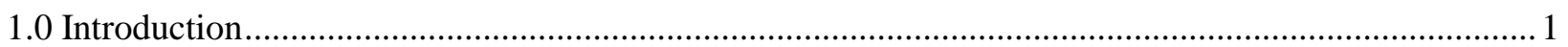

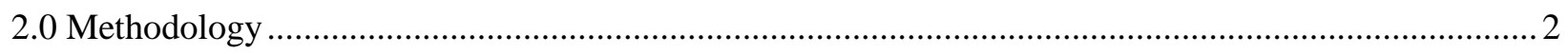

2.1 Each Source Sample Maps to Only One Composite Sample ....................................................... 3

2.2 Each Source Sample Can Map to One or More Composite Samples............................................... 5

3.0 Selection of the Number of Source Samples from the Floor Stratum for each Composite Sample ....... 6

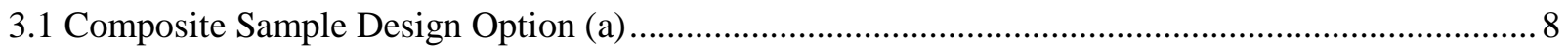

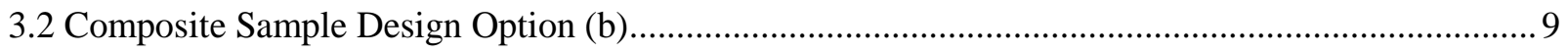

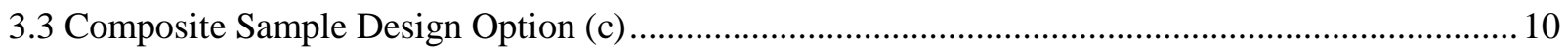

3.4 Comparison of Composite Sample Design Options ........................................................................ 13

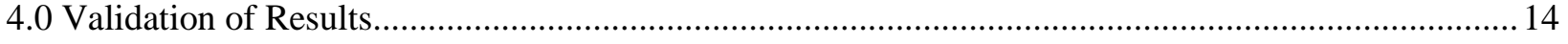

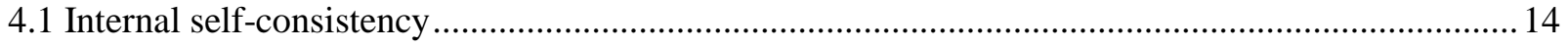

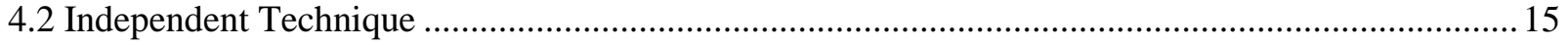

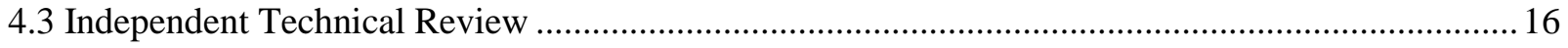

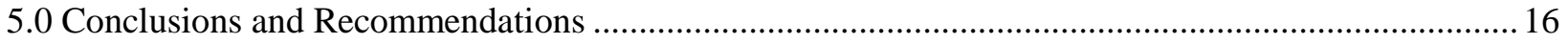

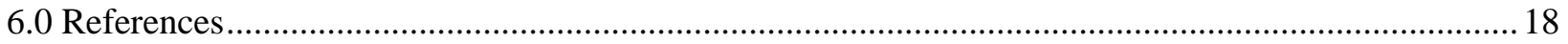




\section{LIST OF TABLES}

Table 1. Indicator Function (Truth Table) for the Expression "Source Sample $j$ from the Low Elevation Mound $\left(M n d_{L}\right)$ Stratum is Assigned to Composite Sample $i$ " and for the Expression "Source Sample $j$ from the High Elevation Mound $\left(M n d_{H}\right)$ Stratum is Assigned to Composite Sample $i$ ”.

Table 2. Indicator Function (Truth Table) for the Expression "Source Sample $j$ from the Floor (Flr) Stratum is Assigned to Composite Sample $i$ ” for Composite Sample Design Option (a) .................... 8

Table 3. Indicator Function (Truth Table) for the Expression "Source Sample $j$ from the Floor (Flr) Stratum is Assigned to Composite Sample $i$ ” for Composite Sample Design Option (b) ................... 10

Table 4. Indicator Function (Truth Table) for the Expression "Source Sample $j$ from the Floor (Flr) Stratum is Assigned to Composite Sample $i$ ” for Composite Sample Design Option (c) .................. 11

Table 5. Indicator Function (Truth Table) for the Expression "Source Sample $j$ from the Floor (Flr) Stratum is Assigned to Composite Sample $i$ ” for an Alternative Strategy for Composite Sample

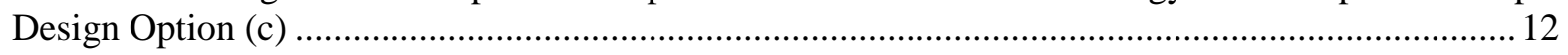

Table 6. Comparison of the Composite Sample Design Options.............................................................. 14

Table 7. Comparison of Simulation Results and Analytical Results ..................................................... 16

\section{LIST OF FIGURES}

Figure 1. Relationships between Source Samples and Composite Samples ............................................. 2

Figure 2. Source Sample Assignment from the Low Elevation Mound Stratum and the High Elevation Mound Stratum to Each Composite Sample...................................................................................... 7

Figure 3. Example of One Source Sample from the Floor Stratum Assigned to Each Composite Sample. 8

Figure 4. Example of One Shared Source Sample from the Floor Stratum Assigned to Two Composite Samples. 10

Figure 5. Example of 3 Floor Stratum Source Samples per Composite Sample: 4 of the 5 Floor Stratum Source Samples Are Shared among 2 Composite Samples.............................................................. 11

Figure 6. Example of 3 Floor Stratum Source Samples per Composite Sample: 3 of the 5 Floor Stratum Source Samples Are Shared among at least 2 Composite Samples. 12 


\section{LIST OF EQUATIONS}

Eqn (1) The True Mean Concentration in the Residual Material in Tank 12Error! Bookmark not defined.

Eqn (2) The True Mean Concentration in the Residual Material in Composite Sample $i, \quad i=1,2, \ldots, N_{c m p}$ Error! Bookmark

Eqn (3) The Statistical Distribution for the Sampling Error/Spatial Heterogeneity in Source Sample $j$

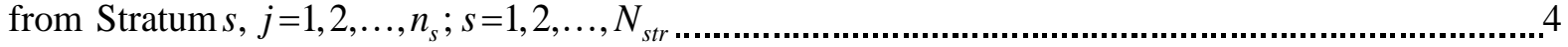

Eqn (4) The Sum over All Composite Sample (true) Concentrations ....................................................

Eqn (5) The True Mean Concentration in the Composite Samples ........................................................ 4

Eqn (6) The Variance of the True Mean Concentration in the Composite Samples.................................. 4

Eqn (7) Definition of an Indicator Function ............................................................................

Eqn (8) A Model for the True Mean Concentration in the Residual Material in Composite Sample $i$, $i=1,2, \ldots, N_{\text {cmp }}$, when Composite Samples Share Source Sample Material .........................................5

Eqn (9) The Sum over All Composite Sample (true) Concentrations when Composite Samples

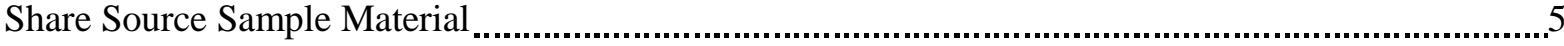

Eqn (10) The True Mean Concentration in the Composite Samples when Composite Samples

Share Source Sample Material 6

Eqn (11) The Variance of the True Mean Concentration in the Composite Samples when Composite Samples Share Source Sample Material 6

Eqn (12) The Variance of the True Mean Concentration in the Composite Samples from the

LWTRSAPP Composite Sample Design 6

Eqn (13) The Variance of the True Mean Concentration in the Composite Samples from the Basic Composite Sample Design 6

Eqn (14) Expanded Form of Eqn (11)

Eqn (15) The Variance of the True Mean Concentration in the Composite Samples from Composite Sample Design Option (a)

Eqn (16) The Variance of the True Mean Concentration in the Composite Samples from Composite Sample Design Option (b)

Eqn (17) The Variance of the True Mean Concentration in the Composite Samples from Composite

Sample Design Option (c) 11 


\section{LIST OF EQUATIONS (CONTINUED)}

Eqn (18) The Variance of the True Mean Concentration in the Composite Samples from

Composite Sample Design Option (c) using an Alternative Configuration

Eqn (19) Sum of Indicator Functions when there is No Sharing of Source Sample Material among Composite Samples,

Eqn (20) The Variance of the True Mean Concentration in the Composite Samples when there is No Sharing of Source Sample material among the Composite Samples.

Eqn (21) Sum of Indicator Functions when there is All of the Source Sample Material among All of the Composite Samples.

Eqn (22) The Variance of the True Mean Concentration in the Composite Samples when All of the Source Sample Material is Shared among All of the Composite Samples

Eqn (23) Variance of a Stratified Random Sample Mean ....................................................................16

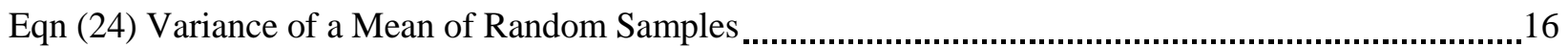




\section{LIST OF ABBREVIATIONS}

$\alpha_{j(s)}$

$\mu$

$\mu_{s}$

LWTRSAPP

$N_{c m p}$

$N_{\text {str }}$

$n_{s}$

TTR

$Y_{i}$

$Y$

$\bar{Y}$

$w_{s}$
The sampling/spatial heterogeneity error for Source Sample $j$ from Stratum $s, j=1,2, \ldots, n_{s} ; s=1,2, \ldots, N_{\text {str }}$.

The true mean concentration in the primary vessel.

The true mean concentration in Stratum $s$ in the primary vessel; $s=1,2, \ldots, N_{s t r}$.

Liquid Waste Tank Residuals Sampling and Analysis Program Plan.

Number of composite samples.

Number of strata.

Number of source samples per composite sample from Stratum $s$, $s=1,2, \ldots, N_{s t r}$.

Task Technical Report.

The true mean concentration in Composite Sample $i, i=1,2, \ldots, N_{c m p}$.

The total of the true mean concentrations in all of the Composite Samples $i, i$ $=1,2, \ldots, N_{\text {cmp }}$. This is an intermediate quantity. The dot subscript indicates that the summation is over the subscript (i) that was replaced by the dot.

The mean of the true mean analyte concentrations in all of the Composite Samples $i, i=1,2, \ldots, N_{\text {cmp }}$.

The relative volume of residual material in Stratum $s, s=1,2, \ldots, N_{\text {str }}$. 


\subsection{Introduction}

The sampling of the residual material from the primary vessel of Tank 12 has been completed. The original sample composite design, Pavletich (2014a), was based on 3 strata: the cooling coils, the floor, and the low and high elevations of an undisturbed mound. A decision was made to address the coiling coils separately from the floor and the mound. The reworked plan, referred to as the Base Composite Sample Design in this report, had 3 composite samples, assigning 2 source samples from the floor, 1 source sample from the low elevation of the mound, and 1 source sample from the high elevation of the mound. Source samples are samples of residual material obtained directly from the tank. This contrasts with composite samples that are not obtained directly from the tank but are constructed by aliquoting material from the source samples. Each of the 12 planned source samples in the Base Composite Sample Design was to contribute residual material to only 1 of the composite samples.

Since only 5 of the 6 floor source samples yielded appreciable material, Pavletich (2014b), the Base Composite Sample Design must be revised. Only "balanced" composite sample designs are considered in this report, where balanced refers to having the same number of source sample contributors for each composite sample. Three options to revise the composite sample design are considered in this report.

Composite Sample Design Option (a). Assign only 1 source sample from the floor stratum and 1 source sample from each of the mound strata to each of the composite samples. Each source sample contributes material to only 1 composite sample. Two source samples would be unused.

Composite Sample Design Option (b). Assign 2 source samples from the floor stratum and 1 source sample from each of the mound strata to each of the composite samples. This infers that one source sample from the floor stratum must be used twice, with 2 composite samples sharing material from this particular source sample. All five source samples from the floor would be used.

Composite Sample Design Option (c). Assign 3 source samples from the floor stratum and 1 source sample from each of the mound strata to each of the composite samples. This infers that several of the 5 source samples from the floor stratum must be used twice. All five source samples from the floor would be used.

This report calculates the mean of the simple average of the composite sample analyte concentrations under two cases:

(1) The source samples are only assigned to a single composite sample.

(2) Source samples can be assigned to more than one composite sample.

Case (1) applies to the Composite Sample Design Option (a), and Case (2) applies to Composite Sample Design Options (b) and (c). The recommendations of this report will be based on an examination of the variance of the mean of the composite samples for Composite Sample Design Options (a), (b), and (c).

This report is based on a revision of the Task Technical Request (TTR) for Tank 12, Pavletich (2014b). 


\subsection{Methodology}

Section 2.0 provides expressions for the true mean analyte concentration and its variance when the composite samples have been constructed from a stratified random sampling design. The true mean concentration in a set of composite samples differs from the true mean concentration in the target region of a tank because of sampling errors and, potentially, spatial heterogeneity within strata. Measurement errors are omitted from the models in this report since all sampling options have an identical measurement error structure. Finite population correction factors, explained in Cochran (1976) and Sukhatme and Sukhatme (1970), are also omitted because the amount of sampled residual material is a small fraction of the material in the target region of Tank 12.

Subsection 2.1 determines the variance of the mean of the composite samples when each source sample contributes residual material to one composite sample. This is depicted for a hypothetical arrangement of 12 source samples and 3 composite samples in Plot (a) of Figure 1. This is the customary method of assigning material from source samples to composite samples. Subsection 2.2 determines the variance of the mean of the composite samples when at least one source sample contributes residual material to two or more composite samples. This is depicted for a hypothetical arrangement of 11 source samples and 3 composite samples in Plot (b) of Figure 1 where a source sample labeled "8” contributes residual material to Composite Samples B and C. This case leads to a correlation between Composite Samples B and C because of the shared material from Source Sample 8. While the mean of the composite samples is unbiased in both cases, there is a weighted mean concentration ${ }^{2}$, Serfling (1980), for the latter case that is unbiased and leads to a smaller variance.

Assessment of plans for revising the design of the composite sample plan will be based on minimizing the (unweighted) variance of composite samples.
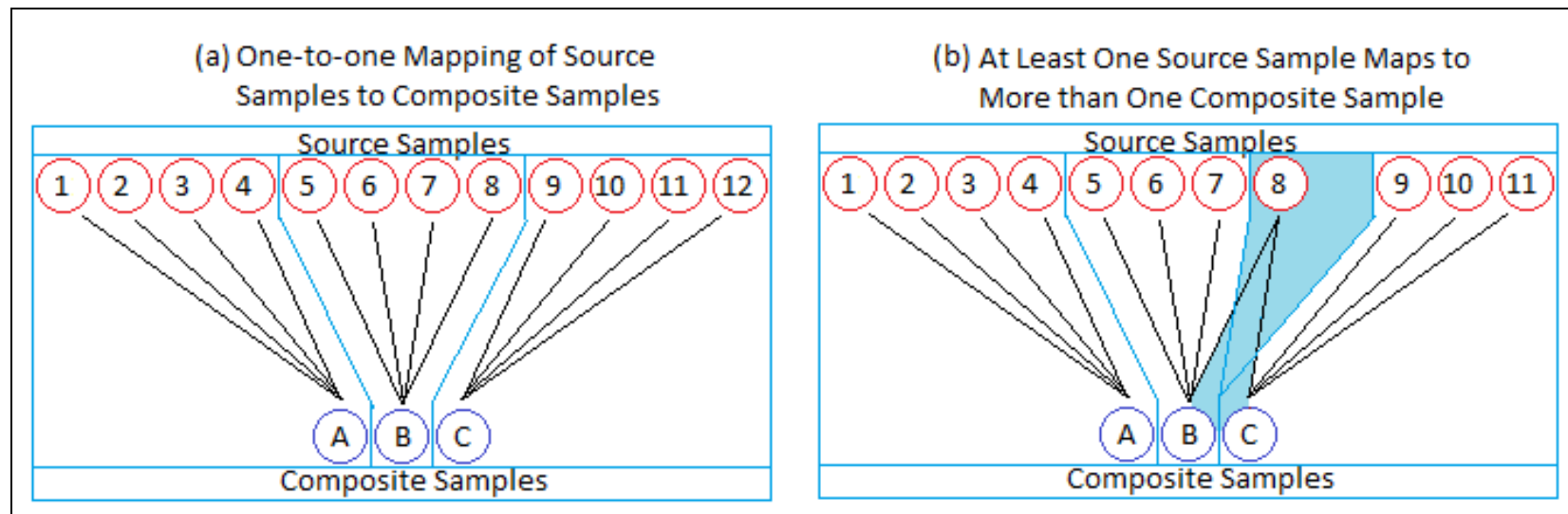

Figure 1. Relationships between Source Samples and Composite Samples

\footnotetext{
2 The weighted mean concentration is not discussed in this report.
} 


\subsection{Each Source Sample Maps to Only One Composite Sample}

This subsection provides an expression for the sampling/spatial heterogeneity variance when each source sample contributes residual material to only one composite sample. The number of composite samples is $N_{c m p}$, and the number of strata is $N_{s t r}$. The number of source samples obtained from Stratum $s$ for each composite sample is $n_{s}, s=1,2, \ldots, N_{s t r}$. Note that the number of source samples from a stratum is the same for all composite samples. Since each source sample is assumed to contribute residual material to only one composite sample, the total number of source samples that must be obtained from the sampling campaign is $N_{c m p} \cdot \sum_{s=1}^{N_{s t r}} n_{s}$. The Base Composite Sample Design had $N_{c m p}=3$ composite samples with $n_{s}=3$ source samples in the floor stratum $(s=F l r)$ and $n_{s}=1$ each of the high elevation and low elevation mound $\operatorname{strata}^{3}$ ( $s=M n d_{H}$ and $M n d_{L}$, respectively), so the total number of originally planned source samples from the primary vessel was $3(2+1+1)=12$.

The true mean concentration in the material in stratum $s$ is $\mu_{s}, s=1,2, \ldots, N_{s t r}$, and the true mean concentration in the residual material left in the entire target area of the tank is given by Eqn (1).

$$
\mu=\sum_{s=1}^{N_{s t r}} w_{s} \mu_{s}
$$

where $w_{s}$ is the relative volume for Stratum $s, s=1,2, \ldots, N_{s t r}$. A model for the true concentration $Y_{i}$ in the residual material in Composite Sample $i, i=1,2, \ldots, N_{c m p}$, is given by Eqn (2).

$$
\begin{array}{r}
Y_{i}=\sum_{s=1}^{N_{s t r}} w_{s}\left(\mu_{s}+\frac{\sum_{j=\left(n_{s}(i-1)+1\right)}^{\left(n_{s} \bullet i\right)} \alpha_{j(s)}}{n_{s}}\right)=\mu+\sum_{s=1}^{N_{s t r}} \frac{w_{s}}{n_{s}} \sum_{j=\left(n_{s}(i-1)+1\right)}^{\left(n_{s} \cdot i\right)} \alpha_{j(s)}, \\
\text { for Composite Sample } i=1,2, \ldots, N_{c m p},
\end{array}
$$

where $\alpha_{j(s)}$ is a random effect ${ }^{4}$ for sampling error/spatial heterogeneity in Source Sample $j$ from Stratum $s$, and it follows the statistical distribution defined in Eqn (3).

\footnotetext{
${ }^{3}$ Technically, the Base Composite Sample Design had 2 strata, the floor and the mound regions, since the cooling coil material was excluded. The mound stratum was configured into low elevation and high elevation substrata. Rather than introducing additional complexity to track strata and substrata, this report took the perspective that the Basic Composite Sample Design effectively had 3 strata: the floor, the low elevation of the mound, and the high elevation of the mound.

${ }^{4}$ In Eqn (2), the notation for the indices in the summation for $j$ enforces the rule that no source sample is shared among the composite samples. For Composite Sample $i=1$, the beginning index is $j=n_{s}(i-1)+1=1$ and the ending index is $j=n_{s} \bullet i=n_{S}$. For Composite Sample $i=2$, the beginning index is $j=n_{s}(i-1)+1=n_{s}+1$ and the ending index is $j=n_{s} \bullet i=2 n_{s}$. For last Composite Sample $i=N_{c m p}$, the beginning index is $j=n_{s}(i-1)+1=n_{s}\left(N_{c m p}-1\right)+1$ and the ending index is $j=n_{s} \bullet i=N_{c m p} \bullet n_{s}$. When $N_{c m p}=3$ composite samples, this last beginning index is $j=n_{s}(i-1)+1=2 n_{s}+1$ and the last ending index is $j=n_{s} \bullet i=3 n_{s}$.
} 
$\alpha_{j(s)} \sim$ Gaussian; $\quad E\left\{\alpha_{j(s)}\right\}=0 ; \quad V\left\{\alpha_{j(s)}\right\}=\sigma_{s}^{2}$, and

$\operatorname{Cov}\left\{\alpha_{j(s)}, \alpha_{j^{\prime}\left(s^{\prime}\right)}\right\}\left\{\begin{array}{l}=0, \text { if either Source Sample } j \neq \text { Source Sample } j^{\prime} \text { when Stratum } s=\text { Stratum } s^{\prime} \\ \quad \text { or Stratum } s \neq \text { Stratum } s^{\prime} . \\ =\sigma_{s}^{2}, \text { where Source Sample } j=\text { Source Sample } j^{\prime} \text { if Stratum } s=\text { Stratum } s^{\prime} .\end{array}\right.$

for Source Samples $j, j^{\prime}=1,2, \ldots, n_{s}$ and Strata $s, s^{\prime}=1,2, \ldots, N_{s t r}$.

Since the expectation of $\alpha_{j(s)}$ is zero, $j=1,2, \ldots, n_{s,} s=1,2, \ldots, N_{s t r}, Y_{i}$ is an unbiased estimator of $\mu$, $i=1,2, \ldots, N_{c m p}$. Every composite sample concentration is an unbiased estimator of the concentration over the entire target area of Tank 12. Summing across all composite samples in Eqn (2) yields Eqn (4).

$$
\begin{aligned}
Y_{\bullet} & =\sum_{i=1}^{N_{c m p}} Y_{i}=\sum_{i=1}^{N_{c m p}}\left[\mu+\sum_{s=1}^{N_{s t r}} \frac{w_{s}}{n_{s}} \sum_{j=\left(n_{s}(i-1)+1\right)}^{\left(n_{s} \cdot i\right)} \alpha_{j(s)}\right] \\
& =N_{c m p} \mu+\sum_{i=1}^{N_{c m p}} \sum_{s=1}^{N_{s t r}} \frac{w_{s}}{n_{s}} \sum_{j=\left(n_{s}(i-1)+1\right)}^{\left(n_{s} \bullet i\right)} \alpha_{j(s)} .
\end{aligned}
$$

Eqn (5) is the true mean concentration in the material over all composite samples. It is obtained by dividing Eqn (4) by the number of composite samples $N_{c m p}$. Note that Eqn (5) is an estimate for the entire target region of the tank, not an exact expression for the true mean in the entire target region of the tank: that is given by Eqn (1).

$$
\overline{Y_{\bullet}}=\frac{Y_{\bullet}}{N_{c m p}}=\frac{N_{c m p} \mu+\sum_{i=1}^{N_{c m p}} \sum_{s=1}^{N_{s t r}} \frac{W_{s}}{n_{s}} \sum_{j=\left(n_{s}(i-1)+1\right)}^{\left(n_{s} \bullet i\right)} \alpha_{j(s)}}{N_{c m p}}=\mu+\frac{1}{N_{c m p}} \sum_{i=1}^{N_{c m p}} \sum_{s=1}^{N_{s t r}} \frac{W_{s}}{n_{s}} \sum_{j=\left(n_{s}(i-1)+1\right)}^{\left(n_{s} \bullet i\right)} \alpha_{j(s)} .
$$

The variance of the true mean of the residual material in the composite samples is given in Eqn (6). This expression applies when each source sample contributes material to only one composite sample. This is the expression for the variance of the mean of independent stratified (composite) sample means, where the variance of a single stratified mean is found in textbooks such as Cochran (1976) and Sukhatme and Sukhatme (1970).

$$
\begin{aligned}
V\{\bar{Y} \bullet\} & =V\left\{\frac{1}{N_{c m p}} \sum_{i=1}^{N_{c m p}} \sum_{s=1}^{N s t r} \frac{w_{s}}{n_{s}} \sum_{\left(n_{s}(i-1)+1\right)}^{\left(n_{s} \cdot i\right)} \alpha_{j(s)}\right\}=\frac{1}{N_{c m p}^{2}} \sum_{i=1}^{N_{c m p}} \sum_{s=1}^{N s t r}\left(\frac{w_{s}}{n_{s}}\right)^{2} \sum_{j=\left(n_{s}(i-1)+1\right)}^{\left(n_{s} \cdot i\right)} \sigma_{s}^{2} \\
& =\frac{1}{N_{c m p}^{2}} \sum_{i=1}^{N_{c m p}} \sum_{s=1}^{N s t r}\left(\frac{w_{s}}{n_{s}}\right)^{2} \sigma_{s}^{2}\left[\left(n_{s} \cdot i\right)-\left(\left(n_{s}(i-1)+1\right)-1\right)\right]=\frac{1}{N_{c m p}^{2}} \sum_{i=1}^{N_{c m p}} \sum_{s=1}^{N s t r}\left(\frac{w_{s}}{n_{s}}\right)^{2} \sigma_{s}^{2} n_{s} \\
& =\frac{1}{N_{c m p}} \sum_{s=1}^{N s t r} w_{s}^{2} \frac{\sigma_{s}^{2}}{n_{s}} .
\end{aligned}
$$




\subsection{Each Source Sample Can Map to One or More Composite Samples}

Subsection 2.2 provides a broader expression for the sampling error/spatial heterogeneity variance of the true mean concentration in the composite samples than the last subsection. Unlike the development in the last subsection, this subsection permits, but does not require source samples to contribute material to more than one composite sample. This sharing of source sample material induces a correlation between the composite samples that receive material from the same source sample(s).

Let $n_{s}$ be the number of source samples from Stratum $s$ that is assigned to each composite sample. Now $N_{c m p} \cdot n_{s}$ is the total number of source samples required for all $N_{c m p}$ composite samples. Let $n_{\text {distinct(s) }}$ be the total number of distinct source samples (that are used in a composite sample design) from Stratum $s$, $s=1,2, \ldots, N_{s t r}$. Note that $n_{\text {distinct(s) }} \leq N_{c m p} \bullet n_{s}$, and $n_{\text {distinct(s) }}$ are the number of distinct source samples from Stratum $s$ that are used in a composite sample design, not the total number of distinct source samples from Stratum s that have been obtained from Tank 12. Also note that $n_{\text {distinct(s) }}=N_{c m p} \bullet n_{s}$, only if all source samples are assigned to only 1 composite sample.

The notation of Subsection 2.1 is cumbersome to perform variance computations when source samples are shared among composite samples, so a different notational scheme is adopted for use here. An indicator function is defined in Eqn (7) to identify if a source sample contributes material to a particular composite sample.

$$
I_{\{\text {Statement }\}}=\left\{\begin{array}{l}
1, \text { if }\{\text { Statement }\} \text { is a true expression, and } \\
0, \text { otherwise, }
\end{array}\right.
$$

where Statement can be any logical expression where the condition "True" is assigned the value 1 and the condition "False" is assigned the value 0 . The particular logical expression " $j(s) \subset i$ " is used as a shortcut notation for "Source Sample $j$ within Stratum $s$ belongs to Composite Sample $i$ ". A model for the true concentration $Y_{i}$ in the material in Composite Sample $i$ can then be described by Eqn (8).

$$
\begin{array}{r}
Y_{i}=\sum_{s=1}^{N_{s t r}} w_{s}\left(\mu_{s}+\frac{\sum_{j=1}^{n_{\text {distinct }(s)}} I_{\{j(s) \subset i\}} \alpha_{j(s)}}{n_{s}}\right)=\mu+\sum_{s=1}^{N_{s t r}} \frac{w_{s}}{n_{s}} \sum_{j=1}^{n_{\text {distinct }(s)}}\left(I_{\{j(s) \subset i\}} \alpha_{j(s)}\right), \\
\text { Composite Sample } i=1,2, \ldots, N_{c m p},
\end{array}
$$

where $\mu$ was defined in Eqn (1) and the random effect $\alpha_{j(s)}$ was defined in Eqn (3). Summing across all composite samples in Eqn (8) yields Eqn (9). Note that the second line interchanges the order of the summation signs.

$$
\begin{aligned}
Y_{\bullet} & =\sum_{i=1}^{n_{c m p}} Y_{i}=N_{c m p} \mu+\sum_{i=1}^{N_{c m p}} \sum_{s=1}^{N_{s t r}} \frac{w_{s}}{n_{s}} \sum_{j=1}^{n_{\text {distinct }(s)}}\left(I_{\{j(s) \subset i\}} \alpha_{j(s)}\right) \\
& =N_{c m p} \mu+\sum_{s=1}^{N_{s t r}} \frac{w_{s}}{n_{s}} \sum_{j=1}^{n_{\text {distinct }(s)}}\left(\alpha_{j(s)} \sum_{i=1}^{N_{c m p}} I_{\{j(s) \subset i\}}\right)
\end{aligned}
$$


Eqn (10) is the true mean analyte concentration in the residual material over all composite samples. It is obtained by dividing Eqn (9) by the number of composite samples $N_{c m p}$.

$$
\bar{Y}_{\bullet}=\frac{Y_{\bullet}}{N_{c m p}}=\mu+\frac{1}{N_{c m p}} \sum_{s=1}^{N_{s t r}} \frac{W_{s}}{n_{s}} \sum_{j=1}^{n_{\text {distinct }(s)}}\left(\alpha_{j(s)} \sum_{i=1}^{N_{c m p}} I_{\{j(s) \subset i\}}\right)
$$

The variance of the true mean of the residual material in the composite samples is given in Eqn (11).

$$
V\left\{\bar{Y}_{\bullet}\right\}=\frac{1}{N_{c m p}^{2}} \sum_{s=1}^{N_{s t r}}\left(\frac{w_{s}}{n_{s}}\right)^{2} \sigma_{s}^{2} \sum_{j=1}^{n_{\text {distinct }(s)}}\left(\sum_{i=1}^{N_{c m p}} I_{\{j(s) \subset i\}}\right)^{2}
$$

\subsection{Selection of the Number of Source Samples from the Floor Stratum for each Composite Sample}

The variance of the mean of the composite samples in the LWTRSAPP, Pavletich (2013), is based on a 15 sample design with the variance of the composite sample mean. If the number of source samples from the strata is set to $n_{M^{n} d_{L}}=1, n_{M n d_{H}}=1$, and $n_{F l r}=3$ in Eqn (6), then Eqn (12) is obtained.

$$
V\left\{\bar{Y}_{\bullet}\right\}=\frac{1}{N_{c m p}}\left[w_{M n d_{L}}^{2} \frac{\sigma_{M n d_{L}}^{2}}{n_{M n d_{L}}}+w_{M n d_{H}}^{2} \frac{\sigma_{M n d_{H}}^{2}}{n_{M n d_{H}}}+w_{F l r}^{2} \frac{\sigma_{F l r}^{2}}{n_{F l r}}\right]=\frac{1}{3}\left[w_{M n d_{L}}^{2} \frac{\sigma_{M n d_{L}}^{2}}{1}+w_{M n d_{H}}^{2} \frac{\sigma_{M n d_{H}}^{2}}{1}+w_{F l r}^{2} \frac{\sigma_{F l r}^{2}}{3}\right]
$$

The Basic Composite Sample Design implemented from Pavletich (2014a) included 6 source samples from the Floor Stratum. If all 6 of these source samples had appreciable material then the variance of the mean of the 3 composite samples is given by Eqn (13).

$$
V\left\{\bar{Y}_{\bullet}\right\}=\frac{1}{3}\left[w_{M n d_{L}}^{2} \frac{\sigma_{M n d_{L}}^{2}}{1}+w_{M n d_{H}}^{2} \frac{\sigma_{M n d_{H}}^{2}}{1}+w_{F l r}^{2} \frac{\sigma_{F l r}^{2}}{2}\right]=\frac{1}{3}\left[w_{M n d_{L}}^{2} \frac{\sigma_{M n d_{L}}^{2}}{1}+w_{M n d_{H}}^{2} \frac{\sigma_{M n d_{H}}^{2}}{1}+1.5 w_{F l r}^{2} \frac{\sigma_{F l r}^{2}}{3}\right]
$$

The composite sample plans underlying Eqns (12) and (13) cannot be implemented since there are only 5 source samples from the floor that have appreciable material. However, the results for the variance of the composite sample concentrations can be compared in this manner for the following composite sample design options that can be supported by 5 source samples from the floor stratum.

Composite Sampling Design Option (a). Assign only 1 source sample from the floor stratum and 1 source sample from each of the low and high mound strata to each of the composite samples. Each source sample contributes material to only 1 composite sample. Two source samples would be unused.

Composite Sampling Design Option (b). Assign 2 source samples from the floor stratum and 1 source samples from each of the low and high mound strata to each composite sample. This infers that one source sample from the floor stratum must be used twice, with 2 
composite samples sharing material from this particular source sample. All five source samples from the floor would be used.

Composite Sampling Design Option (c). Assign 3 source samples from the floor stratum and 1 source samples from each of the low and high mound strata to each composite sample. A scheme to implement this is to use 4 of the 5 source samples from the floor stratum twice. All five source samples from the floor would be used.

Three source samples were obtained from the low elevation mound stratum, and 3 source samples were obtained from the high elevation mound stratum. The portions of the variance in Eqn (11) that arise from source samples from the low elevation and the high elevation mound strata are the same for each of the composite sampling design options since the options only needed to consider how to allocate source sample from the floor stratum to the composite samples. A particular assignment of source samples from either the low elevation mound stratum or the high elevation mound stratum to the composite samples is depicted in Figure 2, where the check mark indicates that a certain source sample is assigned to a certain composite sample. Other possible source sample assignments for this option can be obtained by randomly permuting the rows and columns in Figure 2.

The scheme in Figure 2 leads to the valuation in Table 1 for the indictor function for the logical expressions " $j\left(M n d_{L}\right) \subset i "$ and $" j\left(M n d_{H}\right) \subset i "$, meaning that Source Sample $j$ within the Low Mound $\left(M n d_{L}\right)$ Stratum belongs to Composite Sample $i$ or that Source Sample $j$ within the High Mound $\left(M n d_{H}\right)$ Stratum belongs to Composite Sample $i$. The indicator function was defined in Eqn (7). The results in Table 1 will be used in Eqn (11) to determine the contributions of the mound strata variances of the composite sample average for each of the composite sample design options.

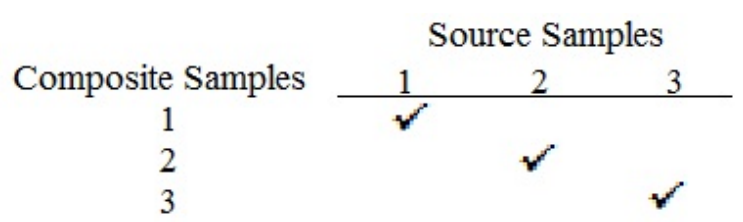

Figure 2. Source Sample Assignment from the Low Elevation Mound Stratum and the High Elevation Mound Stratum to Each Composite Sample

Table 1. Indicator Function (Truth Table) for the Expression "Source Sample $\boldsymbol{j}$ from the Low Elevation Mound $\left(\mathrm{Mnd}_{L}\right)$ Stratum is Assigned to Composite Sample $i$ ” and for the Expression "Source Sample $\boldsymbol{j}$ from the High Elevation Mound $\left(\mathrm{Mnd}_{H}\right)$ Stratum is Assigned to Composite Sample $i$ ”.

\begin{tabular}{|c|c|c|c|}
\hline$I_{\{j(F I r) \subset i\}}$ & \multicolumn{3}{|c|}{ Source Samples } \\
\hline Composite Samples & $j=1\left(M n d_{L}\right)$ or $1\left(M n d_{H}\right)$ & $j=2\left(M n d_{L}\right)$ or $2\left(M n d_{H}\right)$ & $j=3\left(M n d_{L}\right)$ or 3(Mnd $\left.{ }_{H}\right)$ \\
\hline$i=1$ & 1 & 0 & 0 \\
\hline$i=2$ & 0 & 1 & 0 \\
\hline$i=3$ & 0 & 0 & 1 \\
\hline
\end{tabular}




\subsection{Composite Sample Design Option (a)}

This composite sample design assigns 1 distinct source sample from the floor stratum to each of the 3 composite samples. A particular assignment of source samples from the floor stratum to the composite samples is depicted in Figure 3, where the check mark indicates that a certain source sample is assigned to a certain composite sample. Other possible source sample assignments for this option can be obtained by randomly permuting the rows and columns in Figure 3. In Composite Sample Design Option (a) there is no sharing of source sample material among the composite samples, and two source samples labeled 4 and 5 in the figure are not assigned to any of the composite samples.

The scheme in Figure 3 leads to the valuation in Table 2 for the indictor function for the logical expression " $j(F l r) \subset i$ ", meaning Source Sample $j$ within the Floor (Flr) Stratum belongs to Composite Sample $i$. The indicator function was defined in Eqn (7).
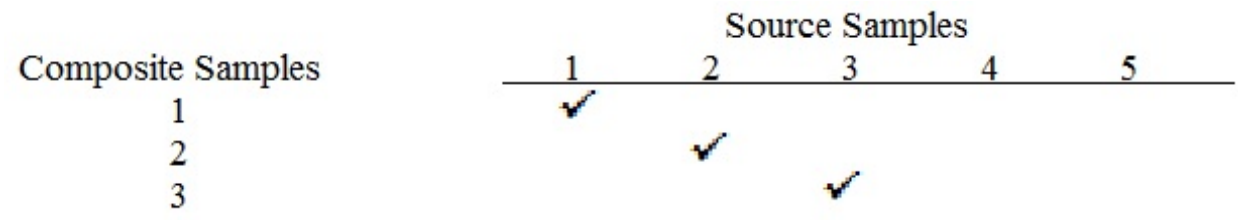

Figure 3. Example of One Source Sample from the Floor Stratum Assigned to Each Composite Sample

Table 2. Indicator Function (Truth Table) for the Expression "Source Sample $\boldsymbol{j}$ from the Floor (Flr) Stratum is Assigned to Composite Sample $i$ ” for Composite Sample Design Option (a)

\begin{tabular}{|c|c|c|c|c|c|}
\hline$I_{\{j(F l r) \subset i\}}$ & \multicolumn{5}{|c|}{ Source Samples } \\
\hline Composite Samples & $j=1($ Flr $)$ & $j=2($ Flr $)$ & $j=3(F l r)$ & $j=4(F l r)$ & $j=5(F l r)$ \\
\hline$i=1$ & 1 & 0 & 0 & 0 & 0 \\
\hline$i=2$ & 0 & 1 & 0 & 0 & 0 \\
\hline$i=3$ & 0 & 0 & 1 & 0 & 0 \\
\hline
\end{tabular}


The variance of $\bar{Y}$. was defined in Eqn (6) and, as a special case, in Eqn (11). Eqn (11) is expanded by stratum in Eqn (14).

$$
\begin{aligned}
V\left\{\bar{Y}_{\bullet}\right\}= & \frac{1}{N_{c m p}^{2}}\left[\left(\frac{w_{M_{n} d_{L}}}{n_{M n d_{L}}}\right)^{2} \sigma_{M n d_{L}}^{2}\left(\left(\sum_{i=1}^{N_{c m p}} I_{\left\{1\left(M n d_{L}\right) \subset i\right\}}\right)^{2}+\left(\sum_{i=1}^{N_{c m p}} I_{\left\{2\left(M n d_{L}\right) \subset i\right\}}\right)^{2}+\left(\sum_{i=1}^{N_{c m p}} I_{\left\{3\left(M n d_{L}\right) \subset i\right\}}\right)^{2}\right)\right. \\
& +\left(\frac{w_{M n d_{H}}}{n_{M n d_{H}}}\right)^{2} \sigma_{M n d_{H}}^{2}\left(\left(\sum_{i=1}^{N_{c m p}} I_{\left\{1\left(M n d_{H}\right) \subset i\right\}}\right)^{2}+\left(\sum_{i=1}^{N_{c m p}} I_{\left\{2\left(M n d_{H}\right) \subset i\right\}}\right)^{2}+\left(\sum_{i=1}^{N_{c m p}} I_{\left\{3\left(M n d_{H}\right) \subset i\right\}}\right)^{2}\right) \\
& +\left(\frac{w_{F l r}}{n_{F l r}}\right)^{2} \sigma_{F l r}^{2}\left(\left(\sum_{i=1}^{N_{c m p}} I_{\{1(F l r) \subset i\}}\right)^{2}+\left(\sum_{i=1}^{N_{c m p}} I_{\{2(F l r) \subset i\}}\right)^{2}+\left(\sum_{i=1}^{N_{c m p}} I_{\{3(F l r) \subset i\}}\right)^{2}\right. \\
& \left.\left.+\left(\sum_{i=1}^{N_{c m p}} I_{\{4(F l r) \subset i\}}\right)^{2}+\left(\sum_{i=1}^{N_{c m p}} I_{\{5(F l r) \subset i\}}\right)^{2}\right)\right]
\end{aligned}
$$

Eqn (15) determines the variance of the mean of the composite samples for Composite Sample Design Option (a) from Eqn (14).

$$
\begin{aligned}
V\left\{\bar{Y}_{\bullet}\right\}=\frac{1}{3^{2}}[( & \left.\frac{w_{M d_{L}}}{1}\right)^{2} \sigma_{M n d_{L}}^{2}\left((1+0+0)^{2}+(0+1+0)^{2}+(0+0+1)^{2}\right) \\
& +\left(\frac{w_{M n d_{H}}}{1}\right)^{2} \sigma_{M n d_{H}}^{2}\left((1+0+0)^{2}+(0+1+0)^{2}+(0+0+1)^{2}\right) \\
& \left.+\left(\frac{w_{F l r}}{1}\right)^{2} \sigma_{F l r}^{2}\left((1+0+0)^{2}+(0+1+0)^{2}+(0+0+1)^{2}+(0+0+0)^{2}+(0+0+0)^{2}\right)\right] \\
= & \frac{1}{9}\left[w_{M n d_{L}}^{2} \frac{3 \sigma_{M n d_{L}}^{2}}{1}+w_{M n d_{H}}^{2} \frac{3 \sigma_{M n d_{H}}^{2}}{1}+w_{F l r}^{2} \frac{3 \sigma_{F l r}^{2}}{1}\right]=\frac{1}{3}\left[w_{M n d_{L}}^{2} \sigma_{M n d_{L}}^{2}+w_{M n d_{H}}^{2} \sigma_{M n d_{H}}^{2}+3 w_{F l r}^{2} \frac{\sigma_{F l r}^{2}}{3}\right]
\end{aligned}
$$

Apart for the leading multiplier, $1 / N_{c m p}=1 / 3$, in the last expression in Eqn (12), the contribution to the variance of the mean of the composite samples from the floor stratum was $w_{F l r}^{2} \sigma_{F l r}^{2} / 3$. The last expression in Eqn (14) has the form of a constant, 3 in this case, times the term $w_{F l r}^{2} \sigma_{F l r}^{2} / 3$ in order to make later composite sample design options comparisons easier.

\subsection{Composite Sample Design Option (b)}

In Composite Sample Design Option (b), at least 1 of the 5 distinct source samples from the floor stratum shares material among the composite samples. A particular assignment of source samples from the floor stratum to the composite samples is depicted in Figure 4 where just 1 of the source samples from the floor stratum shares material with 2 composite samples. A check mark indicates that a certain source sample is assigned to a certain composite sample. Other possible source sample assignments for this option can be obtained by randomly permuting the rows and columns in Figure 4. 
The scheme in Figure 4 leads to the valuation in Table 3 for the indictor function for the logical expression " $j(F l r) \subset i$ ", meaning Source Sample $j$ within the Floor (Flr) Stratum belongs to Composite Sample $i$. The indicator function was defined in Eqn (7).
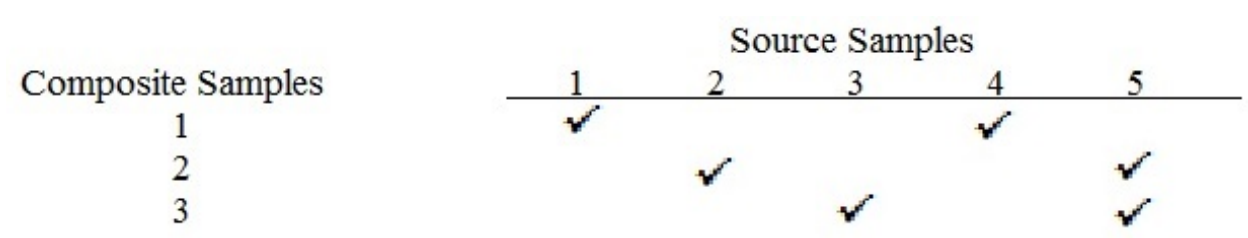

Figure 4. Example of One Shared Source Sample from the Floor Stratum Assigned to Two Composite Samples

Table 3. Indicator Function (Truth Table) for the Expression "Source Sample $\boldsymbol{j}$ from the Floor (Flr) Stratum is Assigned to Composite Sample $i$ ” for Composite Sample Design Option (b)

\begin{tabular}{|c|c|c|c|c|c|}
\hline$I_{\{j(F l r) \subset i\}}$ & \multicolumn{5}{|c|}{ Source Samples } \\
\hline Composite Samples & $j=1($ Flr $)$ & $j=2($ Flr $)$ & $j=3($ Flr $)$ & $j=4($ Flr $)$ & $j=5($ Flr $)$ \\
\hline$i=1$ & 1 & 0 & 0 & 1 & 0 \\
\hline$i=2$ & 0 & 1 & 0 & 0 & 1 \\
\hline$i=3$ & 0 & 0 & 1 & 0 & 1 \\
\hline
\end{tabular}

The variance of $\bar{Y}$. was defined in Eqn (6) and, as a special case, in Eqns (11) and (14). It is evaluated using Eqn (13) for Composite Sample Design Option (b) in Eqn (16).

$$
\begin{aligned}
& V\left\{\bar{Y}_{\bullet}\right\}=\frac{1}{3^{2}}[(\left.\frac{w_{M n d_{L}}}{1}\right)^{2} \sigma_{M n d_{L}}^{2}\left((1+0+0)^{2}+(0+1+0)^{2}+(0+0+1)^{2}\right) \\
&+\left(\frac{w_{M n d_{H}}}{1}\right)^{2} \sigma_{M n d_{H}}^{2}\left((1+0+0)^{2}+(0+1+0)^{2}+(0+0+1)^{2}\right) \\
&\left.+\left(\frac{w_{F l r}}{2}\right)^{2} \sigma_{F l r}^{2}\left((1+0+0)^{2}+(0+1+0)^{2}+(0+0+1)^{2}+(1+0+0)^{2}+(0+1+1)^{2}\right)\right] \\
&=\frac{1}{9}\left[w_{M n d_{L}}^{2} \frac{3 \sigma_{M n d_{L}}^{2}}{1}+w_{M n d_{H}}^{2} \frac{3 \sigma_{M n d_{H}}^{2}}{1}+w_{F l r}^{2} \frac{8 \sigma_{F l r}^{2}}{4}\right]=\frac{1}{3}\left[w_{M n d_{L}}^{2} \sigma_{M n d_{L}}^{2}+w_{M n d_{H}}^{2} \sigma_{M n d_{H}}^{2}+2 w_{F l r}^{2} \frac{\sigma_{F l r}^{2}}{3}\right]
\end{aligned}
$$

\subsection{Composite Sample Design Option (c)}

In Composite Sample Design Option (c), 3 or more source samples from the floor stratum share material among the composite samples. A particular assignment of source samples from the floor stratum to the composite samples is depicted in Figure 5 where 4 of the 5 source samples from the floor stratum share material with 2 composite samples. A check mark indicates that a certain source sample is assigned to a 
certain composite sample. Other possible source sample assignments for this option can be obtained by randomly permuting the rows and columns in Figure 5.

The scheme in Figure 5 leads to the valuation in Table 4 for the indictor function for the logical expression " $j(F l r) \subset i$ ", meaning Source Sample $j$ within the Floor (Flr) Stratum belongs to Composite Sample $i$. The indicator function was defined in Eqn (7).

Composite Samples

$$
\begin{aligned}
& 1 \\
& 2 \\
& 3
\end{aligned}
$$

Figure 5. Example of 3 Floor Stratum Source Samples per Composite Sample: 4 of the 5 Floor Stratum Source Samples Are Shared among 2 Composite Samples

Table 4. Indicator Function (Truth Table) for the Expression "Source Sample $j$ from the Floor (FIr) Stratum is Assigned to Composite Sample $i$ ” for Composite Sample Design Option (c)

\begin{tabular}{|c|c|c|c|c|c|}
\hline$I_{\{j(F l r) \subset i\}}$ & \multicolumn{5}{|c|}{ Source Samples } \\
\hline Composite Samples & $j=1($ Flr $)$ & $j=2($ Flr $)$ & $j=3($ Flr $)$ & $j=4(F l r)$ & $j=5($ Flr $)$ \\
\hline$i=1$ & 1 & 1 & 1 & 0 & 0 \\
\hline$i=2$ & 0 & 0 & 1 & 1 & 1 \\
\hline$i=3$ & 1 & 1 & 0 & 0 & 1 \\
\hline
\end{tabular}

The variance of $\bar{Y}_{\text {. }}$ was defined in Eqn (6) and, as a special case, in Eqns (11) and (13). It is evaluated using Eqn (14) for Composite Sample Design Option (c) in Eqn (17).

$$
\begin{aligned}
V\left\{\bar{Y}_{\bullet}\right\}= & \frac{1}{3_{c m p}^{2}}\left[\left(\frac{w_{M d_{L}}}{1}\right)^{2} \sigma_{M n d_{L}}^{2}\left((1+0+0)^{2}+(0+1+0)^{2}+(0+0+1)^{2}\right)\right. \\
& +\left(\frac{w_{M n d_{H}}}{1}\right)^{2} \sigma_{M n d_{H}}^{2}\left((1+0+0)^{2}+(0+1+0)^{2}+(0+0+1)^{2}\right) \\
& \left.\quad+\left(\frac{w_{F l r}}{3}\right)^{2} \sigma_{F l r}^{2}\left((1+0+1)^{2}+(1+0+1)^{2}+(1+1+0)^{2}+(0+1+0)^{2}+(0+1+1)^{2}\right)\right] \\
= & \frac{1}{9}\left[w_{M n d_{L}}^{2} 3 \sigma_{M n d_{L}}^{2}+3 w_{M n d_{H}}^{2}+w_{F l r}^{2} \frac{17 \sigma_{F l r}^{2}}{9}\right] \\
= & \frac{1}{3}\left[w_{M n d_{L}}^{2} \sigma_{M n d_{L}}^{2}+w_{M n d_{H}}^{2} \sigma_{M n d_{H}}^{2}+1.89 w_{F l r}^{2} \frac{\sigma_{F l r}^{2}}{3}\right]
\end{aligned}
$$


An alternative strategy when 3 source samples from the Floor Stratum are assigned to each composite sample is shown in Figure 6. The scheme in Figure 6 reduces the number of shared source samples from the floor by 1 by increasing the contribution of material from one of the shared source samples to all 3 composite samples. This leads to the valuation in Table 5 for the indictor function for the logical expression " $j(F l r) \subset i$ ", meaning Source Sample $j$ within the Floor (Flr) Stratum belongs to Composite Sample $i$.

Composite Samples
1
2
3

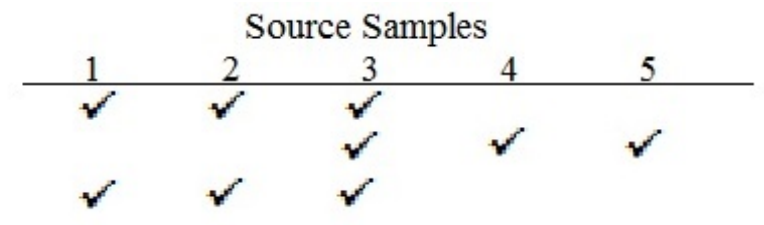

Figure 6. Example of 3 Floor Stratum Source Samples per Composite Sample: 3 of the 5 Floor Stratum Source Samples Are Shared among at least 2 Composite Samples

Table 5. Indicator Function (Truth Table) for the Expression "Source Sample $\boldsymbol{j}$ from the Floor (FIr) Stratum is Assigned to Composite Sample $i$ ” for an Alternative Strategy for Composite Sample Design Option (c)

\begin{tabular}{|c|c|c|c|c|c|}
\hline$I_{\{j(F l r) \subset i\}}$ & \multicolumn{5}{|c|}{ Source Samples } \\
\hline Composite Samples & $j=1($ Flr $)$ & $j=2($ Flr $)$ & $j=3($ Flr $)$ & $j=4($ Flr $)$ & $j=5($ Flr $)$ \\
\hline$i=1$ & 1 & 1 & 1 & 0 & 0 \\
\hline$i=2$ & 0 & 0 & 1 & 1 & 1 \\
\hline$i=3$ & 1 & 1 & 1 & 0 & 0 \\
\hline
\end{tabular}

The variance of $\bar{Y}_{\bullet}$ was defined in Eqn (6) and, as a special case, in Eqns (11) and (14). It is evaluated using Eqn (13) for Composite Sample Design Option (c) in Eqn (18).

$$
\begin{aligned}
& V\left\{\bar{Y}_{\bullet}\right\}=\frac{1}{3_{c m p}^{2}}[\left(\frac{w_{\text {Mnd }_{L}}}{1}\right)^{2} \sigma_{M n d_{L}}^{2}\left((1+0+0)^{2}+(0+1+0)^{2}+(0+0+1)^{2}\right) \\
&+\left(\frac{w_{M_{n d}}}{1}\right)^{2} \sigma_{M n d_{H}}^{2}\left((1+0+0)^{2}+(0+1+0)^{2}+(0+0+1)^{2}\right) \\
&\left.+\left(\frac{w_{F l r}}{3}\right)^{2} \sigma_{F l r}^{2}\left((1+0+1)^{2}+(1+0+1)^{2}+(1+1+1)^{2}+(0+1+0)^{2}+(0+1+0)^{2}\right)\right] \\
&=\frac{1}{9}\left[w_{M n d_{L}}^{2} \frac{3 \sigma_{M n d_{L}}^{2}}{1}+w_{M n d_{H}}^{2} \frac{3 \sigma_{M n d_{H}}^{2}}{1}+w_{F l r}^{2} \frac{19 \sigma_{F l r}^{2}}{9}\right]=\frac{1}{3}\left[w_{\text {Mnd }_{L}}^{2} \sigma_{\text {Mnd }_{L}}^{2}+w_{\text {Mnd }_{H}}^{2} \sigma_{\text {Mnd }_{H}}^{2}+2.11 w_{F l r}^{2} \frac{\sigma_{F l r}^{2}}{3}\right]
\end{aligned}
$$

The contribution from the floor stratum to the variance of $\bar{Y}_{\bullet}$ in Eqn (17) based on the strategy of sharing 3 source samples from the floor stratum is larger than variance of $\bar{Y}$. from Eqn (16) based on the strategy 
of sharing 4 source samples from the floor stratum. This is because the reduction in the number of sources samples being shared by composite sample comes at the expense of sharing one source sample from the floor stratum with all 3 composite samples. So the alternative assignment strategy with one source sample contributing to all 3 composite samples will no longer be considered.

\subsection{Comparison of Composite Sample Design Options}

Each variance formula in Table 6 is structured (from left to right) as the sum of variance contributions from the low elevation of the mound, the high elevation of the mound, and the floor. Since the source sample configurations from the mound strata are the same for all designs in Table 6, the contribution to the variances from the mound strata will be the same. Only the floor stratum contribution will vary by the composite sample design chosen.

This subsection compares the variances for the composite sample mean concentrations for the LWTRSAPP, Pavletich (2013), and the Tank 12 Basic Composite Sample Design with the variances for the Composite Sample Design Options (a), (b), and (c). The top three rows in Table 6 list the variances for the composite sample mean concentrations for the spatial error/spatial heterogeneity for plans having 3 (LWTRSAPP), 2 (Basic Composite Sample Design), and 1 (Composite Sample Design Option (a)) source samples from the floor stratum per composite sample, respectively. All 3 of these designs assign residual material from a source sample to only 1 composite sample. Since the total number of source samples that must be obtained from Tank 12 declines from 15 to 12 to 9 , respectively, the variances for the mean of the composite sample concentrations increase as evidenced by the multiplier (in blue) for the floor sample contribution: 1 to 1.5 to 3 . Since only 5 source samples were obtained from Tank 12, the LWSTSAPP and Basic Composite Sample Designs are not feasible, but offer baselines for comparison.

The variances of the mean concentration $\bar{Y}$. for Composite Sample Design Options (a), (b), and (c) are presented in the shaded area of Table 6. Composite Sample Design Option (a) has the greatest variance of any of the composite sample design options: Option (a) is not recommended. Composite Sample Design Options (b) and (c) offer feasible alternatives to Composite Sample Design Option (a). They differ from these other designs because they allow sharing of source sample material among the composite samples. The smallest variance for the mean concentration in the composite samples is obtained by adopting Composite Sample Design Option (c) which assigns 3 source samples from the Floor Stratum to each composite sample with 4 source samples from the Floor Stratum shared by the composite samples. However, there is little practical difference between the results for Sample Composite Designs (b) and (c). The contributions from the mound strata tend to dilute differences between the contributions from the floor strata. Also, the measurement error, which has not been considered in this report, further moderates differences. 
Table 6. Comparison of the Composite Sample Design Options

\begin{tabular}{|l|l|}
\hline Sample Composite Design & Variance of the Mean of 3 Composite Samples \\
\hline $\begin{array}{l}\text { LWTRSAPP Composite Sample Design } \\
\text { 5 Source Samples for each of 3 Composite Samples. } \\
\text { 3 Source Samples from the Floor Stratum. }\end{array}$ & $V\left\{\bar{Y}_{\bullet}\right\}=\frac{1}{3}\left[w_{M n d_{L}}^{2} \sigma_{M n d_{L}}^{2}+w_{M n d_{H}}^{2} \sigma_{M n d_{H}}^{2}+1 \cdot w_{F l r}^{2} \frac{\sigma_{F l r}^{2}}{3}\right]$ \\
\hline $\begin{array}{l}\text { Tank 12 Basic Composite Sample Design } \\
\text { 4 Source Samples for each of 3 Composite Samples } \\
\text { 2 Source Samples from the Floor Stratum }\end{array}$ & $V\left\{\bar{Y}_{\bullet}\right\}=\frac{1}{3}\left[w_{M n d_{L}}^{2} \sigma_{M n d_{L}}^{2}+w_{M n d_{H}}^{2} \sigma_{M n d_{H}}^{2}+1.5 w_{F l r}^{2} \frac{\sigma_{F l r}^{2}}{3}\right]$ \\
\hline $\begin{array}{l}\text { Feasible Options for Tank 12 when the Basic Composite Sample Design Yields Appreciable Material } \\
\text { in only 5 of the 6 Planned Samples from the Floor Stratum }\end{array}$ \\
$\begin{array}{l}\text { Composite Sample Design Option (a): } \\
\text { 3 Source Samples for each of 3 Composite Samples } \\
\text { 1 Source Sample from the Floor Stratum } \\
\text { 2 Unused Source Samples from the Floor Stratum }\end{array}$ & $V\left\{\bar{Y}_{\bullet}\right\}=\frac{1}{3}\left[w_{M n d_{L}}^{2} \sigma_{M n d_{L}}^{2}+w_{M d_{H}}^{2} \sigma_{M n d_{H}}^{2}+3 w_{F l r}^{2} \frac{\sigma_{F l r}^{2}}{3}\right]$ \\
\hline $\begin{array}{l}\text { Composite Sample Design Option (b): } \\
\text { 4 Source Samples for each of 3 Composite Samples } \\
\text { 2 Source Samples from the Floor Stratum }\end{array}$ & $V\left\{\bar{Y}_{\bullet}\right\}=\frac{1}{3}\left[w_{M n d_{L}}^{2} \sigma_{M n d_{L}}^{2}+w_{M n d_{H}}^{2} \sigma_{M n d_{H}}^{2}+2 w_{F l r}^{2} \frac{\sigma_{F l r}^{2}}{3}\right]$ \\
\hline $\begin{array}{l}\text { Composite Sample Design Option (c): } \\
\text { 5 Source Samples for each of 3 Composite Samples } \\
\text { 3 Source Samples from the Floor Stratum }\end{array}$ & $V\left\{\bar{Y}_{\bullet}\right\}=\frac{1}{3}\left[w_{M n d_{L}}^{2} \sigma_{M n d_{L}}^{2}+w_{M n d_{H}}^{2} \sigma_{M n d_{H}}^{2}+1.89 w_{F l r}^{2} \frac{\sigma_{F l r}^{2}}{3}\right]$ \\
\hline
\end{tabular}

\subsection{Validation of Results}

The variance formulas in Eqn (6) for composite sample designs that do not share source sample material among composite samples and Eqn (11) for composite sample designs that do share source sample material among composite samples have been validated using the following processes based on internal self-consistency, an independent technique to verify numerical results, and an independent technical review.

\subsection{Internal self-consistency}

The following internal consistencies were confirmed. The sum of the values of the indicator function across all composite samples is given in Eqn (6) when there is no sharing of source sample material among composite samples. This situation assigns source samples to only one composite sample, so only one indicator function in the sum in Eqn (19) can equal 1 and all other indicator functions in the sum must be zero.

$$
\sum_{i=1}^{N_{c m p}} I_{\{j(s) \subset i\}}=1,
$$

and $n_{\text {distinct(s) }}=N_{c m p} \bullet n_{s}$ since there is no sharing of material among the composite samples from any source sample. Therefore, Eqn (20) demonstrates that Eqn (11) reduces to Eqn (6) when there is no sharing. 


$$
\begin{aligned}
V\left\{\bar{Y}_{\bullet}\right\} & =\frac{1}{N_{c m p}^{2}} \sum_{s=1}^{N_{s t r}}\left(\frac{w_{s}}{n_{s}}\right)^{2} \sigma_{s}^{2} \sum_{j=1}^{n_{\text {distinct }(s)}}\left(\sum_{i=1}^{N_{c m p}} I_{\{j(s) \subset i\}}\right)^{2}=\frac{1}{N_{c m p}^{2}} \sum_{s=1}^{N_{s t r}}\left(\frac{w_{s}}{n_{s}}\right)^{2} \sigma_{s}^{2} \sum_{j=1}^{n_{\text {distinct }(s)}}(1)^{2} \\
& =\frac{1}{N_{c m p}^{2}} \sum_{s=1}^{N_{s t r}} w_{s}^{2} \frac{\sigma_{s}^{2}}{n_{s}^{2}} n_{\text {distinct }(s)}=\frac{1}{N_{c m p}^{2}} \sum_{s=1}^{N_{s t r}} w_{s}^{2} \frac{\sigma_{s}^{2}}{n_{s}^{2}}\left(N_{c m p} \bullet n_{s}\right)=\frac{1}{N_{c m p}} \sum_{s=1}^{N_{s t r}} w_{s}^{2} \frac{\sigma_{s}^{2}}{n_{s}}
\end{aligned}
$$

In the other extreme, suppose that every source sample from the floor stratum was shared with every composite sample. Then every indicator function has the value one, and the sum of the indicator functions across composite samples is given in Eqn (21).

$$
\sum_{i=1}^{N_{c m p}} I_{\{j(s) \subset i\}}=N_{c m p},
$$

Therefore, Eqn (22) demonstrates that Eqn (11) reduces to Eqn (6) with $N_{c m p}=1$ composite sample when there is no sharing.

$$
\begin{aligned}
V\{\bar{Y} .\} & =\frac{1}{N_{c m p}^{2}} \sum_{s=1}^{3}\left(\frac{w_{s}}{n_{\text {distinct }(s)}}\right)^{2} \sigma_{s}^{2} \sum_{j=1}^{n_{\text {distinct }(s)}}\left(\sum_{i=1}^{N_{c m p}} I_{\{j(s) \subset i\}}\right)^{2} \\
& =\frac{1}{N_{c m p}^{2}} \sum_{s=1}^{3}\left(\frac{w_{s}}{n_{\text {distinct }(s)}}\right)^{2} \sigma_{s}^{2} \sum_{j=1}^{n_{\text {distinct }(s)}}\left(N_{c m p}\right)^{2} \\
& =\frac{1}{N_{c m p}^{2}} \sum_{s=1}^{3}\left(\frac{w_{s}}{n_{\text {distinct }(s)}}\right)^{2} \sigma_{s}^{2} n_{\text {distinct (s) }}\left(N_{c m p}\right)^{2}=\sum_{s=1}^{3} w_{s}^{2} \frac{\sigma_{s}^{2}}{n_{\text {distinct }(s)}}
\end{aligned}
$$

\subsection{Independent Technique}

A simulation was performed with Wolfram Mathematica Version 9.0.1 software, Wolfram Research (2013), that confirmed the numerical results in Table 6. For each iteration, standard normal random deviates (random numbers from a normal distribution with mean 0 and standard deviation 1) were independently generated for source samples from the floor stratum, and combined to produce values for the composite samples based on the specific composite sample design. For 10,000 iterations the variances were computed, and the ratio of the floor stratum contribution to the variance of a particular composite sample design to that of the floor stratum contribution of the Original Composite Sample Design with 9 random source samples from the floor stratum were computed. The ratio results of the simulation are presented in Table 7, and compare to the results from Table 6. 
SRNL-STI-2014-00551

Revision 1

Table 7. Comparison of Simulation Results and Analytical Results

\begin{tabular}{|c|c|c|}
\hline Composite Sample Design Option & Ratio from Simulation & $\begin{array}{c}\text { Leading Constant from the Floor } \\
\text { Sample Term from Table 6 }\end{array}$ \\
\hline (a) & 3.03276 & 3. \\
\hline (b) & 2.00965 & 2. \\
\hline (c) & 1.89268 & 1.88889 \\
\hline
\end{tabular}

\subsection{Independent Technical Review}

The independent technical review had two aspects: a comparison to previous published results and a review by an independent reviewer.

Eqn (6), based on compositing source samples, can be compared to the formula for the variance of a stratified sample average when the source samples are to be separately evaluated (not composited). The latter formula, found in Cochran (1976) and Sukhatme and Sukhatme (1970), is given in Eqn (23). The finite population correction factor was ignored in Eqn (6) and Eqn (23) because the sampled material was small relative to the material in Tank 12.

$$
V\left\{Y_{i}\right\}=\sum_{s=1}^{N_{s t r}} w_{s}^{2} \frac{\sigma_{s}^{2}}{n_{s}} .
$$

The variance, Roussas (1973), of the mean of independent estimators, $Y_{i}, 1,2, \ldots, N_{c m p}$, is given by Eqn (24).

$$
V\left\{\bar{Y}_{.}\right\}=\frac{1}{N_{c m p}} V\left\{Y_{i}\right\}
$$

Substituting Eqn (22) into Eqn (23) produces Eqn (6).

The final draft of this report was submitted to an independent technical reviewer who corroborated the results and signed the approval page upon completion of the technical review.

\subsection{Conclusions and Recommendations}

The SRR sampling campaign to obtain residual solids material from the Savannah River Site (SRS) Tank Farm Tank 12 primary vessel resulted in obtaining appreciable material in all 6 planned source ${ }^{5}$ samples from the mound strata but only in 5 of the 6 planned source samples from the floor stratum. Consequently, the design of the compositing scheme presented in the Tank 12 Sampling and Analysis Plan, Pavletich (2014a), must be revised.

The Analytical Development Directorate of SRNL statistically evaluated the sampling uncertainty associated with using various compositing arrays and splitting one or more samples for compositing. The

\footnotetext{
${ }^{5}$ In this report a source sample is a sample of residual material obtained directly from the primary vessel of Tank 12 . This contrasts with a composite sample which is not obtained directly from Tank 12, but receives aliquots of residual material from some of the source samples.
} 
variance of the simple average of composite sample analyte concentrations is a reasonable standard to investigate the impact of the following sampling options.

Composite Sample Design Option (a). Assign only 1 source sample from the floor stratum and 2 source samples from the mound stratum to each of the composite samples. Each source sample contributes material to only 1 composite sample. Two source samples would be unused.

Composite Sample Design Option (b). Assign 2 source samples from the floor stratum and 2 source samples from the mound stratum to each composite sample. This infers that one source sample from the floor must be used twice, with 2 composite samples sharing material from this particular source sample. All five source samples from the floor would be used.

Composite Sample Design Option (c). Assign 3 source samples from the floor stratum and 2 source samples from the mound stratum to each composite sample. This infers that 4 of the 5 source samples from the floor stratum must be used twice. It will be assumed that each source sample from the floor stratum would be assigned to no more than 2 composite samples. All 5 source samples from the floor would be used.

Using fewer than 12 source samples will increase the sampling variability over that of the Basic Composite Sampling Design. Considering the impact to the variance of the mean concentration in the composite samples, the recommendation is to construct each sample composite using four or five source samples. Although the variance using 5 source samples (Composite Sample Design Option (c)) was slightly less than the variance using 4 source samples (Composite Sample Design Option (b)), there is no practical difference between those variances. This does not consider that the measurement error variance, which is the same for all composite sample design options considered in this report will further dilute any differences. Composite Sample Design Option (a) had the largest variance for the mean of three composite samples and should be avoided.

Once a particular number of source samples per stratum to assign to each composite sample has been determined, sample composite designs that share source sample material with 2 composite samples generally have a smaller variance for the mean of the composite samples than designs that share material among all 3 composite samples.

These results are consistent with Pavletich (2014b) which utilizes a low elevation and a high elevation mound source sample and two floor source samples for each composite sample. Utilizing the four source sample per composite design, Pavletich (2014b) utilizes aliquots of Floor Sample 4 for two composite samples. 


\subsection{References}

(1976) Cochran, W.G. Sampling Techniques. Second Edition. New York, NY: John Wiley \& Sons, Inc.

(2013) Pavletich, J.P. "Liquid Waste Tank Residuals Sampling and Analysis Program Plan,” SRR Interoffice Memorandum SRR-CWDA-2011-00050, Rev. 2. Aiken, SC: Savannah River Remediation (SRR), LLC.

(2014a) Pavletich, J.P., “Tank 12 Sampling and Analysis Plan,” SRR-LWE-2014-00074, Savannah River Site, Aiken, SC, Rev. 0, May 27.

(2014b) Pavletich, J.P. “Tank 12 Technical Task Request Appendix D,” G-TTR-H-00008, Rev. 2.

(1973) Roussas, G.G. A First Course in Mathematical Statistics, Addison-Wesley Publishing Company, Reading, MA.

(1980) Serfling, Robert J. Approximation Theorems of Mathematical Statistics. New York, NY: John Wiley \& Sons, Inc.

(1970) Sukhatme, P.V., and Sukhatme, B.V. Sampling Theory of Surveys with Applications, Second, Revised Edition. Ames, IA: Iowa State University Press.

(2013) Wolfram Mathematica Version 9.0.1. Licensed Software from Wolfram Research, Champaign, IL. 